Supporting Information

\title{
Selection of Ionic Liquids to be used as Separation Agents for Terpenes and Terpenoids
}

\author{
Mónia A. R. Martins ${ }^{1,2}$, Urszula Domańska ${ }^{2}$, Bernd Schröder ${ }^{1}$, João A. P. Coutinho ${ }^{1}$ and \\ Simão P. Pinho ${ }^{3 *}$
}

${ }^{1}$ Departamento de Química, CICECO, Universidade de Aveiro, 3810-193 Aveiro, Portugal.

${ }^{2}$ Physical Chemistry Department, Warsaw University of Technology, 00-661 Warsaw, Poland.

${ }^{3}$ Associate Laboratory LSRE/LCM, Departamento de Tecnologia Química e Biológica, Instituto Politécnico de Bragança, 5301-857 Bragança, Portugal.

*Corresponding author: Phone: +351 273303086; Fax:+351 273313051; E-mail: spinho@ipb.pt.

Number of pages: 21

Number of tables: 4

Number of figures: 3 


\section{Experimental Section}

Chemicals. The individual samples of the ILs used were purified under vacuum $(0.1 \mathrm{~Pa}$ and 353 $\mathrm{K}$ ) and constant stirring, for at least $48 \mathrm{~h}$. The purity was then analysed using ${ }^{1} \mathrm{H}$, ${ }^{13} \mathrm{C}$, and ${ }^{19} \mathrm{~F}$ NMR spectra. In order to further reduce the water traces and the volatile impurities, ILs individual samples were additionally dried during 72 hours at $300 \mathrm{~K}$ under reduced pressure. Karl-Fischer titration was used to determine the water content of the dry ILs. For that, samples were dissolved in dry methanol and titrated with a step of $2.5 \mu \mathrm{L}$. The water content was found to be below 300 ppm for all IL samples. Terpenes and terpenoids were used without any further purification since the GLC technique operates at elevated temperatures $(>398.15 \mathrm{~K})$ and thus, impurities can be removed from the column.

Apparatus and Experimental Procedure. The infinite dilution activity coefficients were estimated from retention times measurements performed using a PerkinElmer Clarus 500 gas chromatograph through the GLC method. Experimental data were collected and treated using the TotalChrom Workstation software. The chromatograph injector and detector were kept at 473.15 $\mathrm{K}$ during the experiments. The carrier gas chosen was helium since it gives greater sensitivity to the TCD detector. The exit gas flow rate and the outlet pressure, $P_{0}$, were both measured with one Agilent Precision Gas Flow Meter placed after the detector, with uncertainties of \pm $0.1 \mathrm{~cm}^{3} \cdot \min ^{-1}$ and $\pm 0.07 \mathrm{kPa}$, respectively. The inlet pressure, $P_{i}$, was measured by the gas chromatograph, through a pressure gauge, with an uncertainty of $\pm 0.1 \mathrm{kPa}$. The settled inlet pressure was $80 \mathrm{kPa}$ while the outlet pressure ranged between [97.84 - 100.18] $\mathrm{kPa}$. The columns were prepared using the rotary evaporation technique, described before. ${ }^{1,2}$ For the packing, between $45 \mathrm{w} \%$ to $55 \mathrm{w} \%$ of the IL stationary phase was used, along with a $100-120$ mesh Chromosorb W/AW - DMCS solid support material (supplied by Sigma-Aldrich). The stationary phase and the solid support were weighed with a precision of $\pm 1 \times 10^{-4} \mathrm{~g}$. High amounts of ILs were utilized in order to prevent the residual adsorption effects of the solutes on the solid support. Methanol was used as solvent in order to promote the ionic liquid coating onto Chromosorb. After that the methanol was evaporated using a vacuum-assisted rotary evaporator and the dry packing was used to fill glass columns of $1 \mathrm{~m}$ length and $4 \mathrm{~mm}$ internal diameter. The final columns were subsequently placed in the chromatograph and a stream of helium gas 
was passed through the stationary phase during $6 \mathrm{~h}(388 \mathrm{~K}$ and $60 \mathrm{kPa})$ for the final drying. Large amounts of column packing are used to avoid the residual adsorption of solute onto the column packing. At least two columns with different packing percentage were prepared for each IL, the repeatability of $\gamma_{13}^{\infty}$ values from two columns were within $\pm 1.5 \%$ for the vast majority of the solutes. Retention times were measured injecting in the column volumes of ( 0.01 to 0.3$) \mu \mathrm{L}$ of the different solutes together with air, a completely non-retained component. Small volumes of solutes were injected to be at infinite dilution conditions. The retention time of air corresponds to the dead time value, $t_{G}$, that was also measured. Each experiment was repeated at least two times in order to check the repeatability that was found to be within $0.01-0.03$ min depending upon the temperature and the solute. The minimum and maximum absolute values of retention times were found to be 0.04 and $249.72 \mathrm{~min}$ corresponding to $\alpha$-pinene and carvacrol, respectively. During the measurements the column temperature was controlled by the gas chromatograph $( \pm 0.02 \mathrm{~K})$. Considering the solute retention times accuracy $( \pm 0.01 \mathrm{~min})$, and the standard deviations (in parentheses) related with: solute vapor pressures $(<5 \%)$, mass of the stationary phase $\left(<1 \times 10^{-4} \mathrm{~g}\right)$, flow rate of helium $\left(0.1 \mathrm{~cm}^{3} \cdot \mathrm{min}^{-1}\right)$, inlet $(0.1 \mathrm{kPa})$ and outlet $(0.07 \mathrm{kPa})$ pressures, and oven temperature $(0.02 \mathrm{~K})$; the uncertainties in $\gamma_{13}^{\infty}$ were estimated by error propagation to be less than $3 \%$.

COSMO-RS. COSMO-RS is a well-stablished a priori method to predict thermophysical properties of fluids and liquid mixtures based on unimolecular quantum calculations. Theoretical details about this method can be found elsewhere. ${ }^{3-5}$ To predict the $\gamma_{13}^{\infty}$ with COSMO-RS first it is necessary to generate distinct input files, for terpenes and terpenoids and IL cations and anions, using BP functional B88-p86 with a triple- $\xi$ valence polarized basis set (TZVP) and the resolution of identity standard (RI) approximation using TURBOMOLE 6.1 program package. $^{6}$ The following calculation consist mainly of statistical thermodynamics and were performed using COSMOtherm, which provides an efficient and flexible implementation of the COSMORS method. The parameterization adopted was BP_TZVP_C30_1401 (COSMOconfX v3.0, COSMOlogic $\mathrm{GmbH} \& \mathrm{Co}$ KG. Leverkusen, Germany). It contains intrinsic parameters of COSMOtherm and element specific parameters, required for the calculation of physiochemical data. In all calculation, ILs have been described by an equimolar mixture of the cation and the anion, that contribute $\sigma$-profile as two different compounds, allowing the study of specific 
contribution of each counter ion. As consequence, it is necessary to scale the calculated values with the factor $0.5 .^{5}$ Moreover, the lowest energy conformations of all the species involved were used in the COSMO-RS calculations.

\section{Theoretical Basis}

In 1960s, Everett ${ }^{7}$ and Cruickshank et al. $^{8}$ developed a relation to calculate the activity coefficient at infinite dilution for a solute (1) partitioning between a carrier gas (2) and a nonvolatile liquid solvent (3) through the solute retention,

$\ln \gamma_{13}^{\infty}=\ln \left(\frac{n_{3} R T}{V_{N} P_{1}^{*}}\right)-\frac{P_{1}^{*}\left(B_{11}-V_{1}^{*}\right)}{R T}+\frac{P_{0} J_{2}^{3}\left(2 B_{12}-V_{1}^{\infty}\right)}{R T}$

where $n_{3}$ refers to the number of moles of solvent (IL) on the column packing, $T$ is the column/oven temperature, $V_{N}$ is the net retention volume of the solute, $P_{1}^{*}$ is the saturated vapour pressure of the solute, $B_{11}$ is the second virial coefficient of the pure solute, $V_{1}^{*}$ is the molar volume of the solute, $P_{0}$ is the outlet pressure, $J_{2}^{3}$ is a pressure correction term, $B_{12}$ is the mixed second virial coefficient of the solute and the carrier gas (helium), and $V_{1}^{\infty}$ is the partial molar volume of the solute at infinite dilution in the solvent. The temperature dependent properties are calculated at $T$. The standard state for the solute is pure liquid at system temperature and zero pressure.

The pressure correction term, $J_{2}^{3},{ }^{9}$ and the net retention volume of the solute, $V_{N}$, are given by:

$$
\begin{aligned}
& J_{2}^{3}=\frac{2}{3} \frac{\left(P_{i} / P_{0}\right)^{3}-1}{\left(P_{i} / P_{0}\right)^{2}-1} \\
& V_{N}=\left(J_{2}^{3}\right)^{-1} U_{0}\left(t_{R}-t_{G}\right)
\end{aligned}
$$

where $t_{R}$ and $t_{G}$ are the retention times for the solute and the unreturned gas (air), respectively. $U_{0}$ is the column outlet volumetric flow rate, corrected for the vapour pressure of water by, 
$U_{0}=U\left(1-\frac{P_{W}}{P_{0}}\right) \frac{T}{T_{f}}$

where $T_{f}$ and $U$ are, respectively, the temperature and the volumetric flow rate, both measured outside the column, and $P_{W}$ is the vapour pressure of water at $T_{f}$.

The properties required for the $\gamma_{13}^{\infty}$ calculations are presented in Table S1, as well as literature sources and methods applied in their estimation. The virial coefficients were calculated using the correlation proposed by Tsonopolous, ${ }^{10}$ available in Poling and Prausnitz. ${ }^{11}$

In order to interpret the interactions and the measured $\gamma_{13}^{\infty}$ data, partial molar excess thermodynamic functions at infinite dilution, namely the Gibbs free energy ( $\bar{G}_{m}^{E, \infty}$ ), enthalpy ( $\left.\bar{H}_{m}^{E, \infty}\right)$ and entropy $\left(\bar{S}_{m}^{E, \infty}\right)$,were calculated through the following equations:

$\bar{G}_{m}^{E, \infty}=R T \ln \left(\gamma_{13}^{\infty}\right)$

$\bar{H}_{m}^{E, \infty}=R\left(\frac{\partial \ln \gamma_{13}^{\infty}}{\partial(1 / T)}\right)_{p, \underline{x}}$

$\bar{S}_{m}^{E, \infty}=\frac{\bar{H}_{m}^{E, \infty}-\bar{G}_{m}^{E, \infty}}{T}$

where subscripts $p$ and $x$ indicate isobaric condition and constant composition, respectively.

Aiming at obtaining information about the recovery of terpenes and terpenoids from ionic liquid mixtures, the gas-liquid partition coefficients $K_{L}=\left(c_{1}^{L} / c_{1}^{G}\right)$ for a solute partitioning between the carrier gas (helium) and the stationary phase (IL), were calculated according to, ${ }^{12}$

$\ln \left(K_{L}\right)=\ln \left(\frac{V_{N} \rho_{3}}{m_{3}}\right)-\frac{P_{0} J_{2}^{3}\left(2 B_{12}-V_{1}^{\infty}\right)}{R T}$ 
being $\rho_{3}$ the density and $m_{3}$ the mass of the IL.

Lastly, to evaluate the performance of ILs as solvents for several practical chemical separation problems, the selectivity between the solutes $i$ and $j, S_{i j}^{\infty}$, and the separation process capacity, $k_{j}^{\infty}$ , are determined as follow: ${ }^{13}$

$S_{i j}^{\infty}=\gamma_{i}^{\infty} / \gamma_{j}^{\infty}$

$k_{j}^{\infty}=1 / \gamma_{j}^{\infty}$

where $j$ is the solute that presents the smaller $\gamma_{13}^{\infty}$ in the solvent (ILs). 
Table S1. Vapor pressures coefficients ${ }^{a}$, densities coefficients ${ }^{b}$, critical properties ${ }^{c}$, acentric factors ${ }^{c}$ and dipole moments ${ }^{d}$ of the terpenes and terpenoids studied.

\begin{tabular}{|c|c|c|c|c|c|c|c|c|c|c|c|c|c|c|c|}
\hline \multirow{3}{*}{ Solutes } & \multicolumn{5}{|c|}{$\frac{\text { Vapor Pressure }}{10^{(\mathrm{A}-\mathrm{B} /(T+\mathrm{C}))}}$} & \multirow{2}{*}{\multicolumn{5}{|c|}{$\frac{\text { Density }}{\rho=\mathrm{A} \cdot T^{2}+\mathrm{B} \cdot T+\mathrm{C}, \rho / \mathrm{kmol} \cdot \mathrm{m}^{-3}, T / \mathrm{K}}$}} & \multicolumn{3}{|c|}{ Critical Properties } & \multirow{3}{*}{$\begin{array}{c}\text { Acentric } \\
\text { Factor } \\
\omega\end{array}$} & \multirow{3}{*}{$\begin{array}{c}\text { Dipole } \\
\text { Moment } \\
\mu \text { / C m }\end{array}$} \\
\hline & & $p=10^{(A}$ & $\mathrm{B} /(T+\mathrm{C})), p / \mathrm{P}$ & $T / \mathrm{K}$ & & & & & & & \multirow{2}{*}{$T_{\mathrm{c}}, \mathrm{K}$} & \multirow{2}{*}{$\mathrm{V}_{\mathrm{c}}, \mathrm{cm}^{3} / \mathrm{mol}$} & \multirow{2}{*}{$\mathrm{P}_{\mathrm{c}}, \mathrm{Pa}$} & & \\
\hline & A & B & $\mathrm{C}$ & $T_{\min }$ & $T_{\max }$ & A & B & $\mathrm{C}$ & $T_{\min }$ & $T_{\max }$ & & & & & \\
\hline$\alpha$-pinene & 9.325 & 1636.919 & -48.140 & 238.19 & 308.15 & $-2.001 \mathrm{E}-06$ & $-4.866 \mathrm{E}-03$ & 7.905 & 278.16 & 368.14 & 630.78 & 484.50 & $2.891 \mathrm{E}+06$ & 0.326 & $1.163 \mathrm{E}-30$ \\
\hline$\beta$-pinene & 9.344 & 1687.684 & -47.835 & 248.13 & 308.15 & $-1.446 \mathrm{E}-06$ & $-5.050 \mathrm{E}-03$ & 7.996 & 278.16 & 368.14 & 646.03 & 482.50 & $2.884 \mathrm{E}+06$ & 0.320 & $4.022 \mathrm{E}-30$ \\
\hline (-)-borneol & 14.261 & 4288.902 & 13.509 & 273.65 & 308.15 & $6.425 \mathrm{E}-07$ & $-7.641 \mathrm{E}-04$ & 6.455 & 473.15 & 523.15 & 670.24 & 514.50 & $3.167 \mathrm{E}+06$ & 0.698 & $7.215 \mathrm{E}-30$ \\
\hline (-)-isopulegol & 9.876 & 1981.013 & -65.493 & 260.00 & 340.00 & $-9.100 \mathrm{E}-07$ & $-4.831 \mathrm{E}-03$ & 7.393 & 278.16 & 368.14 & 656.76 & 527.50 & $2.770 \mathrm{E}+06$ & 0.698 & $9.703 \mathrm{E}-30$ \\
\hline$(-)$-menthone & 8.846 & 1499.364 & -93.462 & 372.00 & 397.00 & $-4.033 \mathrm{E}-07$ & $-4.810 \mathrm{E}-03$ & 7.240 & 278.16 & 368.14 & 689.70 & 528.50 & $2.595 \mathrm{E}+06$ & 0.412 & $1.450 \mathrm{E}-29$ \\
\hline (1R)-(-)-fenchone & 9.684 & 1936.425 & -46.047 & 243.15 & 308.15 & $-5.096 \mathrm{E}-07$ & $-5.228 \mathrm{E}-03$ & 7.784 & 283.16 & 368.14 & 679.18 & 503.50 & $3.083 \mathrm{E}+06$ & 0.388 & $1.466 \mathrm{E}-29$ \\
\hline$(\mathrm{S})-(+)$-carvone & 10.048 & 2364.370 & -31.980 & 320.00 & 511.00 & $-4.877 \mathrm{E}-07$ & $-5.046 \mathrm{E}-03$ & 7.922 & 278.16 & 368.14 & 724.81 & 503.50 & $2.860 \mathrm{E}+06$ & 0.419 & $1.779 \mathrm{E}-29$ \\
\hline Carvacrol & 10.342 & 2549.857 & -32.705 & 343.00 & 510.00 & $-2.735 \mathrm{E}-06$ & $-3.413 \mathrm{E}-03$ & 7.733 & 279.51 & 368.14 & 722.20 & 447.50 & $3.440 \mathrm{E}+06$ & 0.581 & $6.273 \mathrm{E}-30$ \\
\hline DL-citronellol & 9.909 & 2045.850 & -79.149 & 341.98 & 554.17 & $-3.210 \mathrm{E}-06$ & $-2.545 \mathrm{E}-03$ & 6.512 & 279.85 & 368.14 & 657.87 & 589.50 & $2.448 \mathrm{E}+06$ & 0.848 & $7.863 \mathrm{E}-30$ \\
\hline Eucalyptol & 9.382 & 1725.544 & -52.393 & 253.15 & 308.15 & $-9.128 \mathrm{E}-07$ & $-5.033 \mathrm{E}-03$ & 7.550 & 278.16 & 368.14 & 661.05 & 509.50 & $3.019 \mathrm{E}+06$ & 0.339 & $8.034 \mathrm{E}-30$ \\
\hline Eugenol & 10.081 & 2457.820 & -42.580 & 351.00 & 530.00 & $-5.632 \mathrm{E}-07$ & $-5.039 \mathrm{E}-03$ & 8.022 & 278.16 & 368.14 & 735.58 & 444.50 & $3.581 \mathrm{E}+06$ & 0.676 & $1.181 \mathrm{E}-29$ \\
\hline Geraniol & 10.730 & 2773.110 & -18.707 & 277.15 & 503.15 & $-3.161 \mathrm{E}-06$ & $-2.790 \mathrm{E}-03$ & 6.796 & 288.16 & 368.14 & 671.67 & 576.50 & $2.571 \mathrm{E}+06$ & 0.820 & $1.185 \mathrm{E}-29$ \\
\hline $\mathrm{L}(-)$-menthol & 10.383 & 2405.948 & -37.850 & 329.15 & 485.15 & $-5.486 \mathrm{E}-06$ & $-1.239 \mathrm{E}-03$ & 6.578 & 318.16 & 358.14 & 661.63 & 539.50 & $2.660 \mathrm{E}+06$ & 0.716 & $7.842 \mathrm{E}-30$ \\
\hline Linalool & 4.556 & 341.017 & -183.210 & 368.00 & 428.00 & $-3.680 \mathrm{E}-06$ & $-3.230 \mathrm{E}-03$ & 6.852 & 280.01 & 368.14 & 633.30 & 565.50 & $2.582 \mathrm{E}+06$ & 0.755 & $9.673 \mathrm{E}-30$ \\
\hline$\alpha$-pinene oxide & 7.758 & 975.321 & -127.664 & 298.15 & 482.00 & $-9.571 \mathrm{E}-07$ & $-4.906 \mathrm{E}-03$ & 7.861 & 278.16 & 368.14 & 716.40 & 489.50 & $3.093 \mathrm{E}+06$ & 0.369 & $9.757 \mathrm{E}-30$ \\
\hline
\end{tabular}

${ }^{a}$ References: $\alpha$-pinene, ${ }^{14} \beta$-pinene, ${ }^{14}(-)$-borneol,,${ }^{15}(-)$-isopulegol: not publish yet, $(-)$-menthone, ${ }^{16}(1 \mathrm{R})-(-)$-fenchone, ${ }^{15}(\mathrm{R})-(+)$-camphor, ${ }^{17}(\mathrm{~S})-(+)$-carvone,${ }^{18}$ Carvacrol, ${ }^{19}$ DL-citronellol, ${ }^{17}$ Eucalyptol, ${ }^{20}$ Eugenol, ${ }^{18}$ Geraniol,,${ }^{17}$ L(-)-menthol, ${ }^{21}$ Linalool, ${ }^{22}$ Thymol, ${ }^{19}$ a-pinene oxide ${ }^{23-25}{ }^{b}$ Reference ${ }^{26}{ }^{c}$ Estimated using 
the Joback group-contribution method, ${ }^{27,28}{ }^{d}$ Estimated using TURBOMOLE 6.1 programme package on the density functional theory level, utilizing the BP functional B88-P86 with a triple- $\zeta$ valence polarized basis set (TZVP) and the resolution of identity standard (RI) approximation. ${ }^{6}$ 
Table S2. Experimental activity coefficients at infinite dilution of terpenes and terpenoids in ILs, at different temperatures. ${ }^{a}$

\begin{tabular}{|c|c|c|c|c|c|c|c|c|c|c|c|c|}
\hline \multirow[t]{2}{*}{ Solutes } & \multicolumn{6}{|c|}{$\left[\mathrm{C}_{4} \mathrm{mim}\right] \mathrm{Cl}^{b}$} & \multicolumn{6}{|c|}{$\left[\mathrm{C}_{4} \mathrm{mim}\right]\left[\mathrm{CH}_{3} \mathrm{SO}_{3}\right]^{c}$} \\
\hline & 398.15 & 408.15 & 418.15 & 428.15 & 438.15 & 448.15 & 398.15 & 408.15 & 418.15 & 428.15 & 438.15 & 448.15 \\
\hline$\alpha$-pinene & 301.13 & 279.40 & 266.50 & 252.23 & 237.17 & 225.94 & 59.41 & 57.88 & 56.08 & 54.21 & 52.58 & 51.15 \\
\hline$\beta$-pinene & 210.73 & 201.65 & 195.20 & 188.20 & 182.73 & 176.27 & 43.19 & 42.43 & 41.67 & 40.97 & 40.23 & 39.53 \\
\hline (-)-borneol & 3.60 & 3.32 & 3.06 & 2.84 & 2.54 & 2.30 & & & & & & \\
\hline (-)-Isopulegol & 7.87 & 8.42 & 8.97 & 9.34 & 9.88 & 10.22 & 3.78 & 3.98 & 4.13 & 4.29 & 4.40 & 4.52 \\
\hline$(-)$-menthone & 90.84 & 91.53 & 91.08 & 90.86 & 90.42 & 88.74 & 17.33 & 17.46 & 17.44 & 17.39 & 17.37 & 17.40 \\
\hline$(1 \mathrm{R})-(-)$-fenchone & 63.56 & 62.09 & 61.24 & 59.35 & 57.54 & 57.58 & 12.58 & 12.55 & 12.36 & 12.27 & 12.16 & 12.05 \\
\hline$(1 \mathrm{R})-(+)$-camphor & 50.74 & 51.46 & 51.49 & 51.58 & 51.61 & 51.12 & 10.71 & 10.78 & 10.81 & 10.85 & 10.87 & 10.90 \\
\hline$(\mathrm{S})-(+)$-carvone & 37.64 & 38.20 & 38.70 & 38.10 & 38.37 & 38.85 & 9.35 & 9.52 & 9.61 & 9.70 & 9.72 & 9.77 \\
\hline \multicolumn{13}{|l|}{ Carvacrol } \\
\hline DL-citronellol & 7.45 & 8.04 & 8.63 & 9.04 & 9.63 & 10.02 & & & & & & \\
\hline Eucalyptol & 163.23 & 160.88 & 155.07 & 148.60 & 148.80 & 144.22 & 32.35 & 32.02 & 31.68 & 31.14 & 30.76 & 30.25 \\
\hline \multicolumn{13}{|l|}{ Eugenol } \\
\hline Geraniol & 5.26 & 5.84 & 6.44 & 6.92 & 7.51 & 7.94 & & & & & & \\
\hline $\mathrm{L}(-)$-menthol & 9.68 & 10.44 & 11.17 & 11.90 & 12.19 & 13.02 & 4.56 & 4.85 & 5.00 & 5.27 & 5.43 & 5.62 \\
\hline Linalool & 85.73 & 118.63 & 161.79 & 213.13 & 282.51 & 364.59 & 39.49 & 53.93 & 72.09 & 95.84 & 127.35 & 162.26 \\
\hline \multicolumn{13}{|l|}{ Thymol } \\
\hline$\alpha$-pinene oxide & 88.60 & 91.21 & 94.04 & 99.10 & 101.31 & 104.70 & 7.94 & 8.61 & 9.26 & 9.95 & 10.67 & 11.49 \\
\hline
\end{tabular}


Table S2. Activity coefficients at infinite dilution of terpenes and terpenoids in ILs, at different temperatures. ${ }^{a}$ (cont)

\begin{tabular}{|c|c|c|c|c|c|c|c|c|c|c|c|c|}
\hline \multirow{2}{*}{$\begin{array}{c}\text { Solutes } \\
T / \mathbf{K}\end{array}$} & \multicolumn{6}{|c|}{$\left[\mathrm{C}_{4} \mathrm{mim}\right]\left[\left(\mathrm{CH}_{3}\right)_{2} \mathrm{PO}_{4}\right]^{d}$} & \multicolumn{6}{|c|}{$\left[\mathrm{C}_{4} \mathrm{mim}\right]\left[\mathrm{CF}_{3} \mathrm{SO}_{3}\right]^{e}$} \\
\hline & 398.15 & 408.15 & 418.15 & 428.15 & 438.15 & 448.15 & 398.15 & 408.15 & 418.15 & 428.15 & 438.15 & 448.15 \\
\hline$\alpha$-pinene & 29.54 & 28.98 & 28.43 & 28.04 & 27.81 & 27.72 & 25.73 & 25.11 & 24.37 & 23.84 & 23.20 & 22.76 \\
\hline$\beta$-pinene & 23.09 & 22.79 & 22.47 & 22.28 & 22.14 & 22.06 & 19.40 & 18.92 & 18.63 & 18.25 & 17.86 & 17.58 \\
\hline (-)-borneol & & & & & & & 2.80 & 2.45 & 2.12 & 1.86 & 1.66 & 1.41 \\
\hline (-)-Isopulegol & & & & & & & 3.84 & 3.92 & 3.95 & 4.01 & 4.09 & \\
\hline (-)-menthone & 12.41 & 12.44 & 12.52 & 12.67 & 12.86 & 13.13 & 5.87 & 5.96 & 6.06 & 6.17 & 6.28 & 6.47 \\
\hline (1R)-(-)-fenchone & 8.94 & 8.91 & 8.86 & 8.89 & 8.87 & 8.90 & 4.16 & 4.10 & 4.15 & 4.19 & 4.22 & 4.25 \\
\hline$(1 \mathrm{R})-(+)$-camphor & 7.93 & 7.96 & 7.98 & 8.02 & 8.05 & 8.11 & 3.71 & 3.78 & 3.86 & 3.93 & 4.03 & 4.09 \\
\hline$(\mathrm{S})-(+)$-carvone & 7.17 & 7.26 & 7.35 & 7.46 & 7.55 & 7.67 & 4.13 & 4.21 & 4.28 & 4.34 & 4.40 & 4.44 \\
\hline Carvacrol & & & & & & & & 0.60 & 0.66 & 0.73 & 0.81 & 0.86 \\
\hline DL-citronellol & & & & & & & 5.13 & 5.24 & 5.29 & 5.39 & 5.55 & \\
\hline Eucalyptol & 19.04 & 18.81 & 18.91 & 18.88 & 18.70 & 18.91 & 12.34 & 12.31 & 12.33 & 12.34 & 12.38 & 12.32 \\
\hline Eugenol & & & & & & & & 1.25 & 1.34 & 1.45 & 1.55 & 1.59 \\
\hline Geraniol & & & & & & & 4.09 & 4.29 & 4.45 & 4.65 & 4.83 & \\
\hline $\mathrm{L}(-)$-menthol & & & & & & & 5.50 & 5.62 & 5.66 & 5.74 & 5.84 & \\
\hline Linalool & 9.96 & 14.26 & 20.00 & 27.73 & 37.18 & 52.02 & 48.52 & 63.49 & 81.46 & 104.32 & 132.40 & 158.94 \\
\hline Thymol & & & & & & & & 0.60 & 0.67 & 0.75 & 0.82 & 0.88 \\
\hline$\alpha$-pinene oxide & 11.40 & 11.95 & 12.60 & 13.24 & 13.90 & 14.75 & 6.18 & 6.49 & 6.86 & 7.24 & 7.64 & 7.90 \\
\hline
\end{tabular}

${ }^{a}$ Standard uncertainties are: $u\left(\gamma_{13}^{\infty}\right)=3 \%$ and $u(T)=0.02 \mathrm{~K} .{ }^{b}$ Packing: $52.1 \%, n_{3}=17.02$ mmol. ${ }^{c}$ Packing: $49.8 \%, n_{3}=11.48 \mathrm{mmol} .{ }^{d}$ Packing: $47.3 \%, n_{3}=9.39 \mathrm{mmol} .{ }^{e}$ Packing: $49.1 \%, n_{3}=8.75 \mathrm{mmol}$. 
Table S3. Infinite dilution partial molar excess enthalpies $\bar{H}_{m}^{E, \infty} / \mathrm{kJ} \cdot \mathrm{mol}^{-1}$, excess Gibbs free energies $\bar{G}_{m}^{E, \infty} / \mathrm{kJ} \cdot \mathrm{mol}^{-1}$, and entropies $T_{r e f} \bar{S}_{m}^{E, \infty} /$ $\mathrm{kJ} \cdot \mathrm{mol}^{-1}$, for terpenes, terpenoids and water in the ILs investigated in this work, at $408.15 \mathrm{~K}^{a}$

\begin{tabular}{|c|c|c|c|c|c|c|c|c|c|c|c|c|}
\hline \multirow[b]{2}{*}{ Solutes } & \multicolumn{3}{|c|}{$\left[\mathrm{C}_{4} \mathrm{mim}\right] \mathrm{Cl}$} & \multicolumn{3}{|c|}{$\left[\mathrm{C}_{4} \mathrm{mim}\right]\left[\mathrm{CH}_{3} \mathrm{SO}_{3}\right]$} & \multicolumn{3}{|c|}{$\left[\mathrm{C}_{4} \mathrm{mim}\right]\left[\left(\mathrm{CH}_{3}\right)_{2} \mathrm{PO}_{4}\right]$} & \multicolumn{3}{|c|}{$\left[\mathrm{C}_{4} \mathrm{mim}\right]\left[\mathrm{CF}_{3} \mathrm{SO}_{3}\right]$} \\
\hline & $\bar{H}_{m}^{E, \infty}$ & $\bar{G}_{m}^{E, \infty}$ & $T_{r e f} \bar{S}_{m}^{E, \infty}$ & $\bar{H}_{m}^{E, \infty}$ & $\bar{G}_{m}^{E, \infty}$ & $T_{r e f} \bar{S}_{m}^{E, \infty}$ & $\bar{H}_{m}^{E, \infty}$ & $\bar{G}_{m}^{E, \infty}$ & $T_{r e f} \bar{S}_{m}^{E, \infty}$ & $\bar{H}_{m}^{E, \infty}$ & $\bar{G}_{m}^{E, \infty}$ & $T_{r e f} \bar{S}_{m}^{E, o}$ \\
\hline$\alpha$-pinene & 8.41 & 19.11 & -10.70 & 4.54 & 13.77 & -9.24 & 1.94 & 11.42 & -9.48 & 3.70 & 10.94 & -7.24 \\
\hline (-)-borneol & 13.18 & 4.08 & 9.10 & & & & & & & 20.00 & 3.04 & 16.96 \\
\hline (-)-isopulegol & -7.75 & 7.23 & -14.98 & -5.20 & 4.69 & -9.89 & & & & -1.35 & 4.64 & -5.99 \\
\hline (-)-menthone & 0.00 & 15.33 & -15.33 & 0.00 & 9.70 & -9.70 & -1.64 & 8.56 & -10.19 & -2.78 & 6.06 & -8.84 \\
\hline$(\mathrm{S})-(+)$-carvone & 0.00 & 12.36 & -12.36 & -1.25 & 7.65 & -8.90 & -1.97 & 6.73 & -8.70 & -2.18 & 4.88 & -7.06 \\
\hline Carvacrol & & & & & & & & & & -14.14 & -1.74 & -12.40 \\
\hline DL-citronellol & -8.79 & 7.07 & -15.86 & & & & & & & -2.13 & 5.62 & -7.75 \\
\hline Eucalyptol & 3.80 & 17.24 & -13.44 & 2.00 & 11.76 & -9.76 & 0.00 & 9.96 & -9.96 & 0.00 & 8.52 & -8.52 \\
\hline Eugenol & & & & & & & & & & -9.47 & 0.77 & -10.24 \\
\hline Geraniol & -12.26 & 5.99 & -18.25 & & & & & & & -5.05 & 4.94 & -10.00 \\
\hline$\alpha$-pinene oxide & -5.09 & 15.32 & -20.41 & -10.87 & 7.31 & -18.18 & -7.58 & 8.42 & -16.00 & -7.51 & 6.35 & -13.86 \\
\hline
\end{tabular}

${ }^{a}$ Standard uncertainties: $u\left(\bar{H}_{m}^{E, \infty}\right)= \pm 0.5 \mathrm{~kJ} \cdot \mathrm{mol}^{-1} ; u\left(\bar{G}_{m}^{E, \infty}\right)= \pm 0.5 \mathrm{~kJ} \cdot \mathrm{mol}^{-1} ; u\left(T_{r e f} \bar{S}_{m}^{E, \infty}\right)= \pm 0.05 \mathrm{~kJ} \cdot \mathrm{mol}^{-1}$. 
Table S4. Gas-liquid partition coefficients, $K_{L}$, for terpenes, terpenoids and water in ILs, at different temperatures. ${ }^{a}$

\begin{tabular}{|c|c|c|c|c|c|c|c|c|c|c|c|c|c|c|c|c|c|c|c|c|c|c|c|c|}
\hline \multirow[t]{2}{*}{ Solutes } & \multicolumn{6}{|c|}{$\left[\mathrm{C}_{4} \mathrm{mim}\right] \mathrm{Cl}$} & \multicolumn{6}{|c|}{$\left[\mathrm{C}_{4} \mathrm{mim}\right]\left[\mathrm{CH}_{3} \mathrm{SO}_{3}\right]$} & \multicolumn{6}{|c|}{$\left[\mathrm{C}_{4} \mathrm{mim}\right]\left[\left(\mathrm{CH}_{3}\right)_{2} \mathrm{PO}_{4}\right]$} & \multicolumn{6}{|c|}{$\left[\mathrm{C}_{4} \mathrm{mim}\right]\left[\mathrm{CF}_{3} \mathrm{SO}_{3}\right]$} \\
\hline & 398.15 & 408.15 & 418.15 & 428.15 & 438.15 & 448.15 & 398.15 & 408.15 & 418.15 & 428.15 & 438.15 & 448.15 & 398.15 & 408.15 & 418.15 & 428.15 & 438.15 & 448.15 & 398.15 & 408.15 & 418.15 & 428.15 & 438.15 & 448.15 \\
\hline$\alpha$-pinene & 1.50 & 1.23 & 1.00 & 0.83 & 0.71 & 0.60 & 6.12 & 4.78 & 3.82 & 3.11 & 2.55 & 2.12 & 10.81 & 8.38 & 6.61 & 5.27 & 4.23 & 3.43 & 12.58 & 9.80 & 7.81 & 6.26 & 5.12 & 4.22 \\
\hline$\beta$-pinene & 2.83 & 2.23 & 1.77 & 1.43 & 1.16 & 0.97 & 11.08 & 8.50 & 6.64 & 5.26 & 4.23 & 3.45 & 18.19 & 13.89 & 10.80 & 8.48 & 6.74 & 5.42 & 21.96 & 16.95 & 13.18 & 10.47 & 8.44 & 6.86 \\
\hline (-)-borneol & 785.84 & 493.04 & 318.50 & 210.54 & 147.30 & 104.85 & & & & & & & & & & & & & 721.70 & 477.97 & 328.00 & 227.97 & 160.66 & 120.92 \\
\hline (-)-isopulegol & 301.22 & 193.18 & 127.16 & 87.57 & 60.45 & 43.43 & 503.45 & 327.95 & 221.51 & 152.85 & 108.90 & 78.73 & & & & & & & 441.15 & 295.91 & 205.79 & 144.85 & 103.77 & \\
\hline$(-)$-menthone & 25.93 & 18.36 & 13.47 & 10.06 & 7.67 & 6.03 & 109.14 & 77.31 & 56.45 & 42.16 & 32.01 & 24.66 & 133.68 & 95.17 & 68.98 & 50.76 & 37.91 & 28.66 & 286.68 & 201.15 & 144.21 & 105.37 & 78.39 & 58.65 \\
\hline$(1 \mathrm{R})-(-)$-fenchone & 20.49 & 15.15 & 11.31 & 8.75 & 6.88 & 5.32 & 83.16 & 60.19 & 44.97 & 33.95 & 26.08 & 20.37 & 102.66 & 74.40 & 55.03 & 41.12 & 31.37 & 24.17 & 223.61 & 163.73 & 119.02 & 88.16 & 66.51 & 51.02 \\
\hline (R)-(+)-camphor & 42.39 & 30.00 & 21.98 & 16.39 & 12.46 & 9.72 & 161.32 & 115.00 & 84.01 & 62.51 & 47.41 & 36.52 & 191.09 & 136.64 & 99.87 & 74.21 & 56.13 & 43.03 & 414.57 & 291.12 & 208.69 & 153.06 & 113.35 & 86.09 \\
\hline$(\mathrm{S})-(+)$-carvone & 134.12 & 91.00 & 63.12 & 45.91 & 33.23 & 24.34 & 433.81 & 293.06 & 203.87 & 144.70 & 105.21 & 77.48 & 496.30 & 337.15 & 233.97 & 165.02 & 118.77 & 86.61 & 874.47 & 589.10 & 406.25 & 286.47 & 205.67 & 150.81 \\
\hline Carvacrol & & & & & & & & & & & & & & & & & & & & 6714.15 & 4107.89 & 2576.97 & 1645.91 & 1110.90 \\
\hline DL-citronellol & 841.24 & 507.87 & 316.87 & 207.26 & 136.49 & 93.85 & & & & & & & & & & & & & 873.79 & 556.69 & 367.87 & 247.18 & 167.95 & \\
\hline Eucalyptol & 4.97 & 3.74 & 2.93 & 2.35 & 1.83 & 1.50 & 20.14 & 15.09 & 11.53 & 9.01 & 7.11 & 5.72 & 30.03 & 22.55 & 16.94 & 13.03 & 10.26 & 8.03 & 46.97 & 34.89 & 26.29 & 20.15 & 15.65 & 12.43 \\
\hline Eugenol & & & & & & & & & & & & & & & & & & & & 5011.76 & 3157.99 & 2020.42 & 1324.07 & 924.91 \\
\hline Geraniol & 1416.48 & 845.52 & 519.65 & 334.42 & 217.06 & & & & & & & & & & & & & & 1300.25 & 822.09 & 536.47 & 353.62 & 239.69 & \\
\hline $\mathrm{L}(-)$-menthol & 399.55 & 249.86 & 160.94 & 106.33 & 74.34 & 50.84 & 680.85 & 432.01 & 288.46 & 192.58 & 133.78 & 94.33 & & & & & & & 502.93 & 331.35 & 226.39 & 156.75 & 110.18 & \\
\hline Linalool & 244.81 & 153.37 & 98.79 & 66.64 & 45.13 & 31.67 & 426.84 & 270.88 & 177.93 & 118.87 & 80.28 & 57.04 & 1485.84 & 898.76 & 562.53 & 360.36 & 241.14 & 156.00 & 309.23 & 204.54 & 139.79 & 96.84 & 68.37 & 51.50 \\
\hline Thymol & & & & & & & & & & & & & & & & & & & & 5323.24 & 3270.67 & 2061.33 & 1337.80 & 908.40 \\
\hline$\alpha$-pinene oxide & 15.81 & 11.70 & 8.81 & 6.61 & 5.21 & 4.11 & 141.77 & 99.48 & 71.78 & 52.85 & 39.59 & 29.97 & 86.62 & 62.91 & 46.30 & 34.81 & 26.66 & 20.47 & 162.01 & 117.24 & 86.09 & 64.34 & 48.95 & 38.55 \\
\hline
\end{tabular}

${ }^{a}$ Standard uncertainties: $u\left(K_{L}\right)<3 \%$ and $u(T)=0.02 \mathrm{~K}$. 

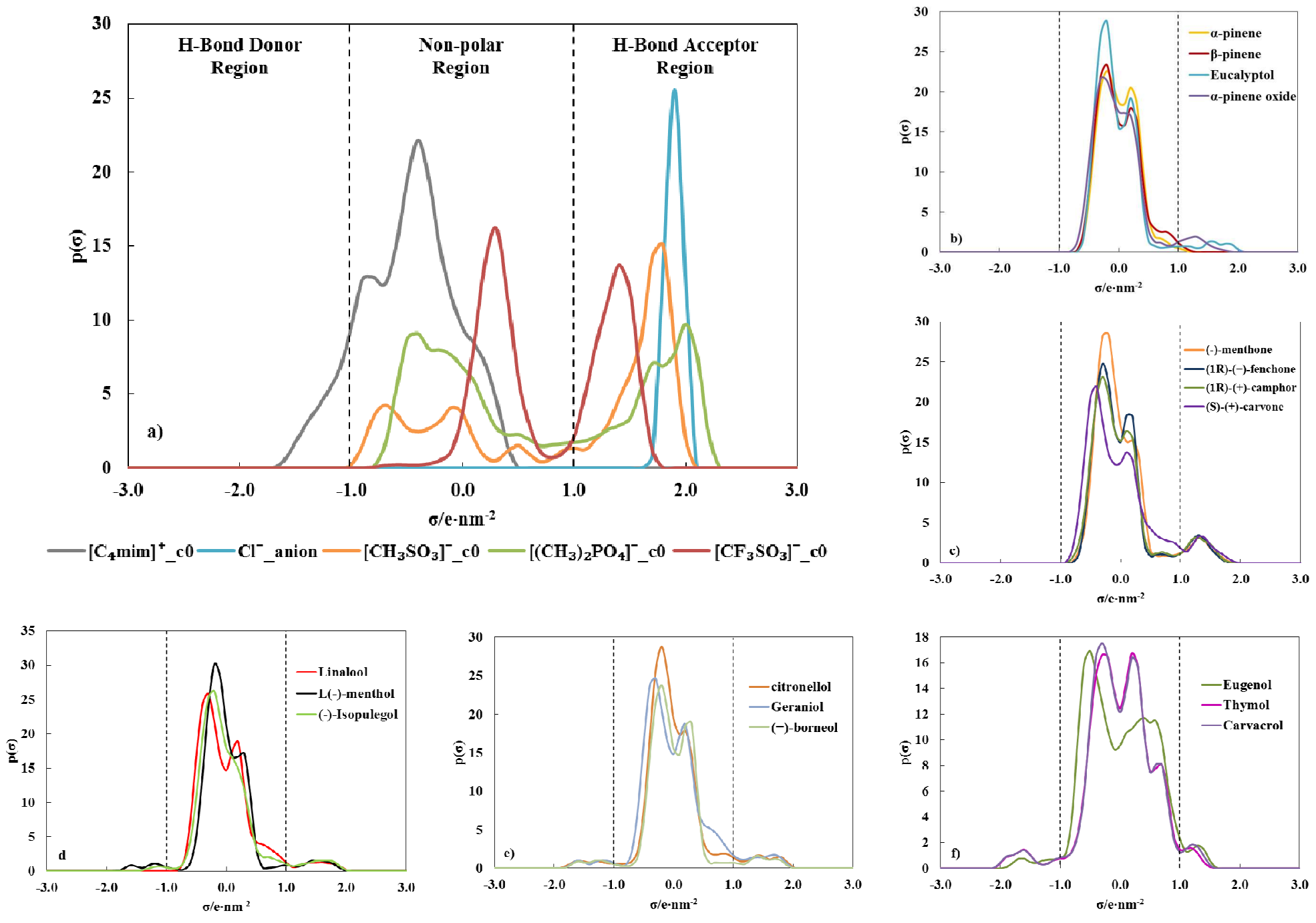
Figure S1. Sigma profiles of: a) ionic liquids; b) hydrocarbons and ethers; c) ketones; d), e) and f) alcohols, computed by COSMO-RS. 

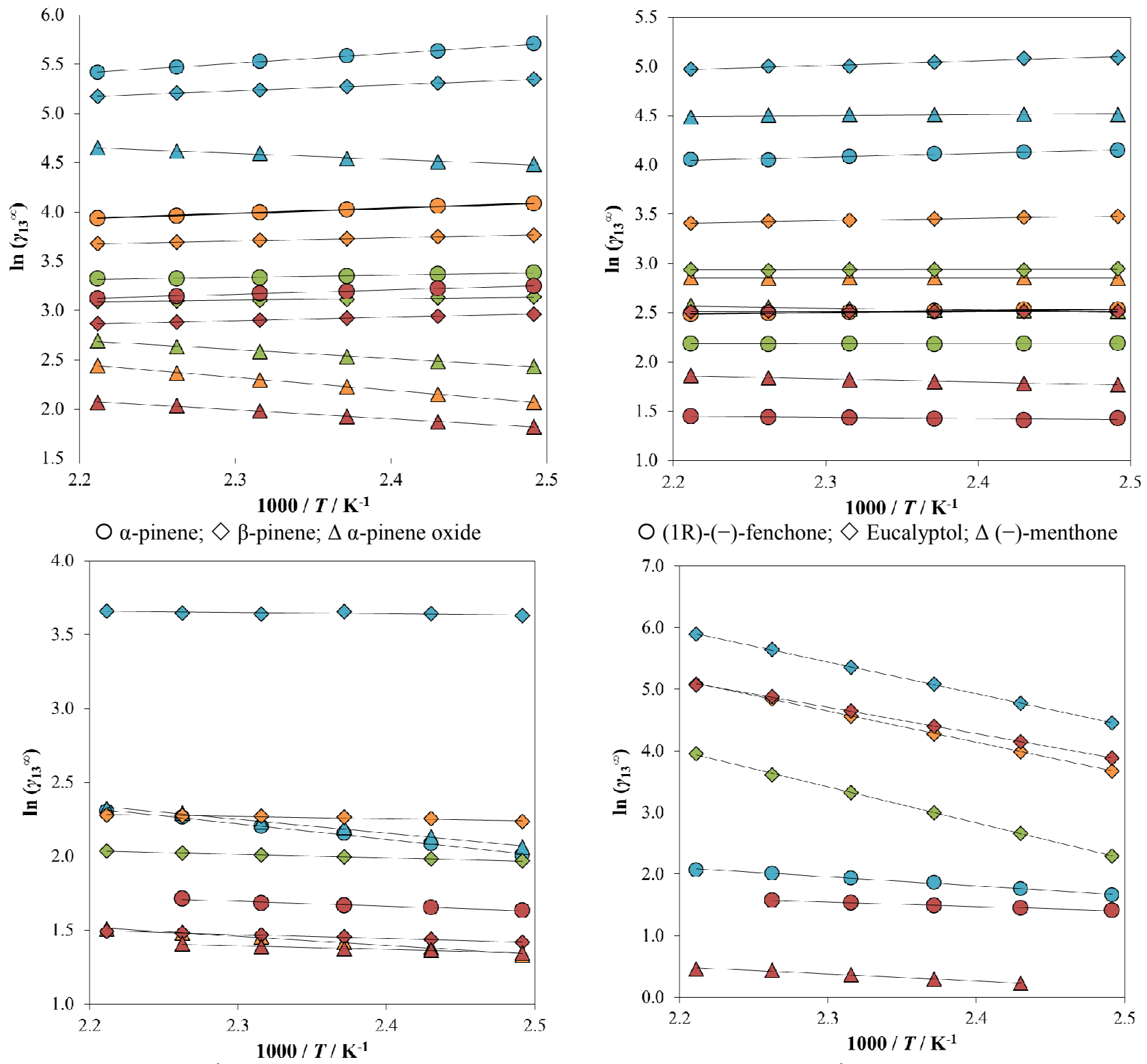

$\bigcirc(1 \mathrm{R})-(-)$-fenchone; $\diamond$ Eucalyptol; $\Delta$ (-)-menthone

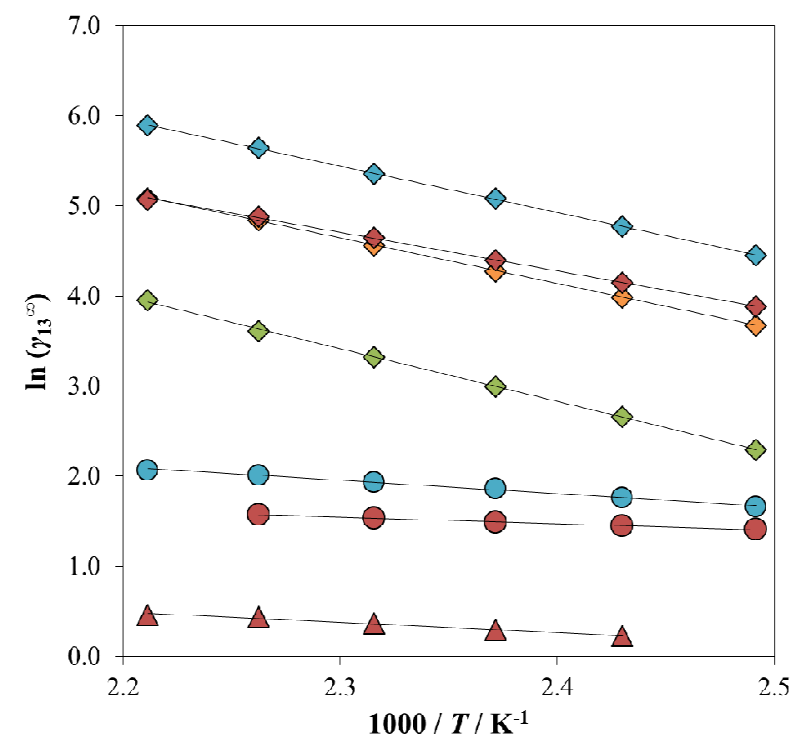

$\bigcirc$ DL-citronellol; $\diamond(\mathrm{S})-(+)$-carvone; $\Delta(-)$-isopulegol 


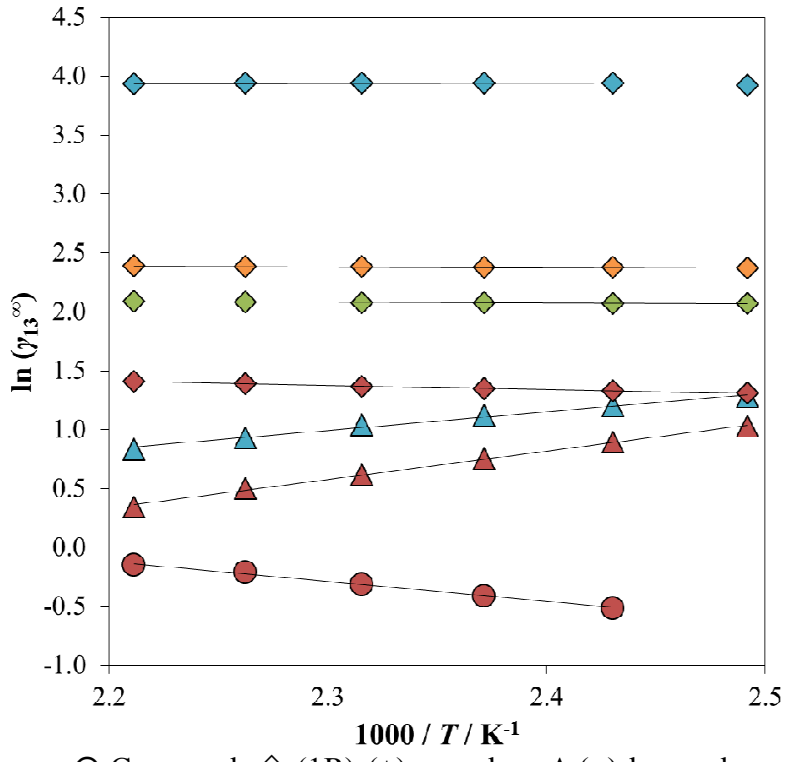

$\bigcirc$ Carvacrol; $\diamond(1 \mathrm{R})-(+)$-camphor; $\Delta(-)$-borneol

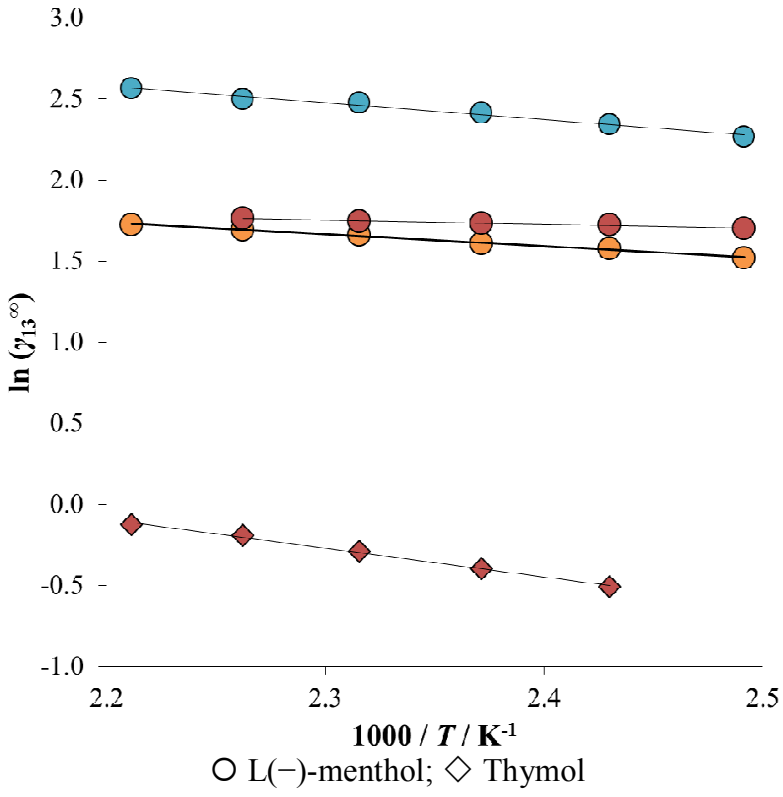

Figure S2. Experimental natural logarithm of activity coefficients at infinite dilution as a function of the inverse absolute temperature for all the studied solutes in: $\bullet$, $\left[\mathrm{C}_{4} \mathrm{mim}\right] \mathrm{Cl} ; \circlearrowleft,\left[\mathrm{C}_{4} \mathrm{mim}\right]\left[\mathrm{CH}_{3} \mathrm{SO}_{3}\right] ; \diamond,\left[\mathrm{C}_{4} \mathrm{mim}\right]\left[\left(\mathrm{CH}_{3}\right)_{2} \mathrm{PO}_{4}\right]$ and $\bullet,\left[\mathrm{C}_{4} \mathrm{mim}\right]\left[\mathrm{CF}_{3} \mathrm{SO}_{3}\right]$. 

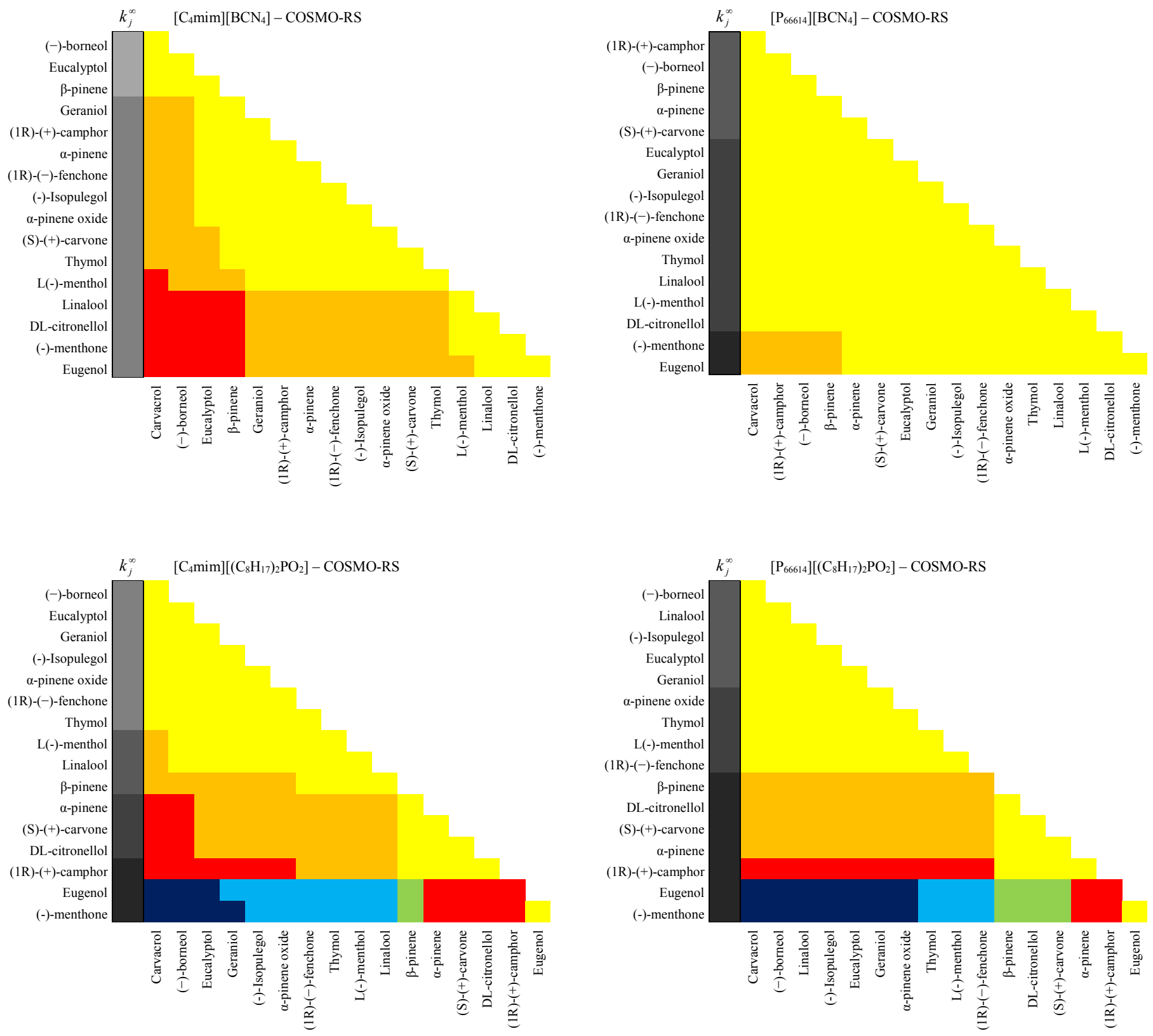

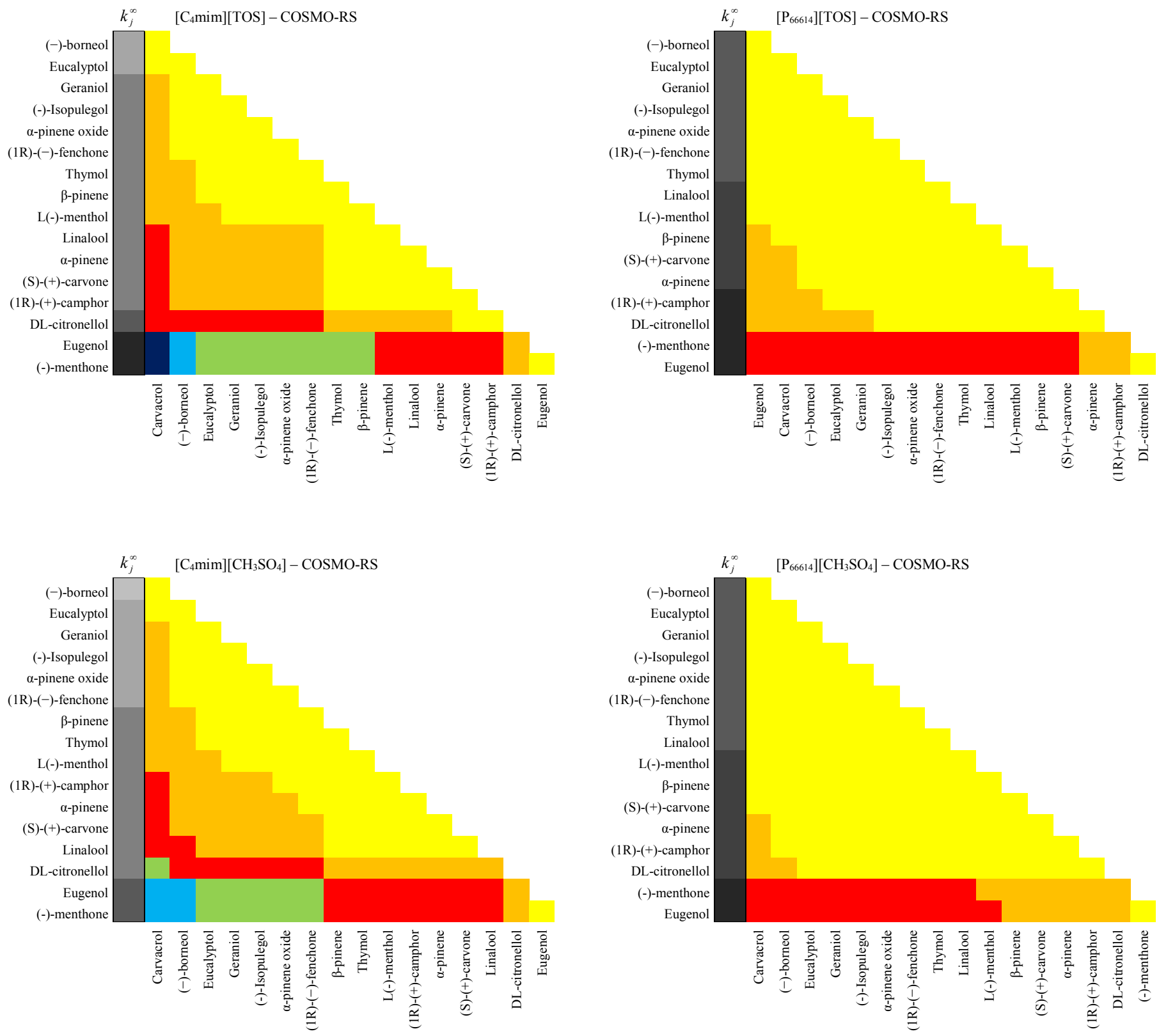

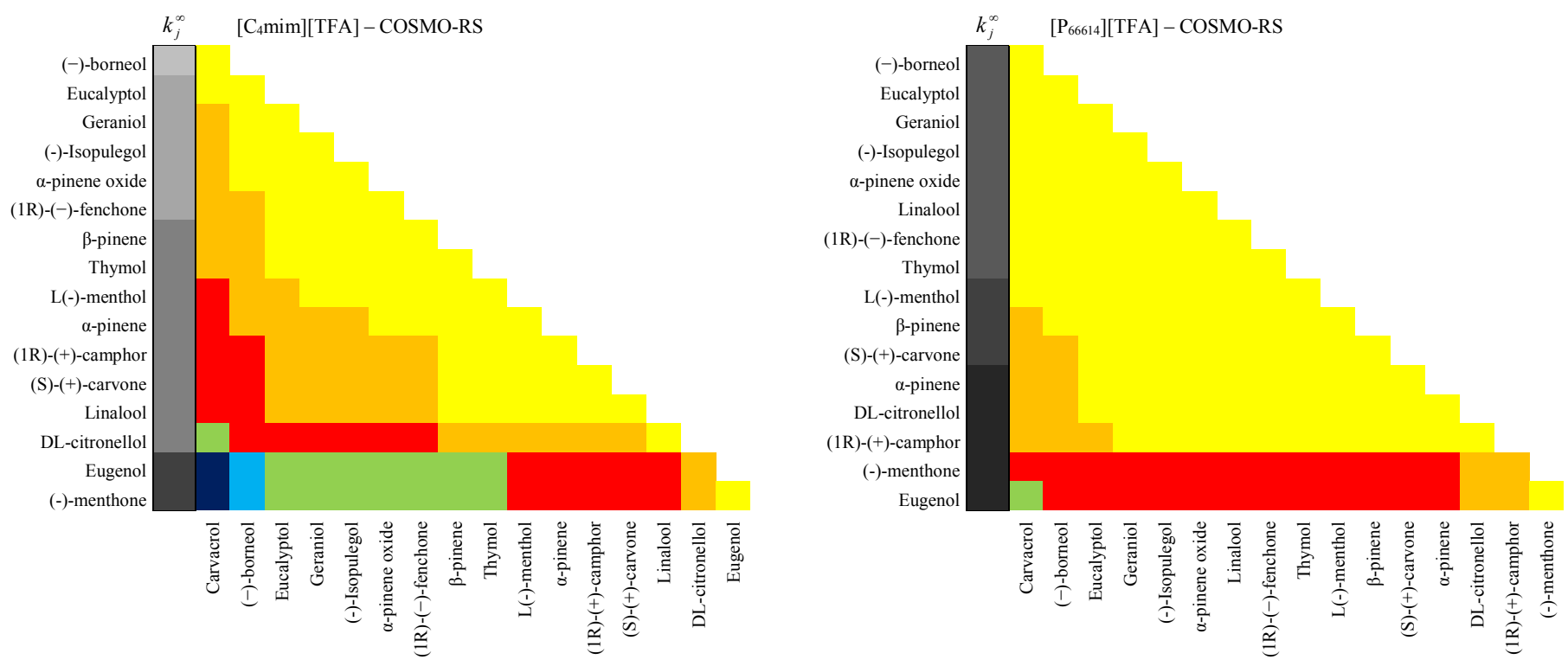

Figure S3. $S_{i j}^{\infty}$ and $k_{j}^{\infty}$ of all solutes at $408.15 \mathrm{~K}$ in selected ionic liquids, computed by COSMO-RS. Cations: $\quad\left[\mathrm{C}_{4} \mathrm{mim}\right]^{+}, \quad$ 1-butyl-3-methyl-imidazolium; $\quad\left[\mathrm{P}_{66614}\right]^{+}$, trihexyltetradecylphosphonium. Anions: $\left[\mathrm{BCN}_{4}\right]^{-}$, tetracyanoborate; $\left[\left(\mathrm{C}_{8} \mathrm{H}_{17}\right)_{2} \mathrm{PO}_{2}\right]^{-}$, bis(2,4,4-trimethylpentyl)phosphinate; [TOS]', tosylate; $\left[\mathrm{CH}_{3} \mathrm{SO}_{4}\right]^{-}$, methylsulfate; and [TFA]', trifluoroacetate. Color code: Selectivities, [1-2]; $\diamond$ [2-4]; $\diamond$ [4-10]; $\diamond[10-20]$; $\diamond[20-30]$; and $\diamond>30$; Capacities, $<0.01 ; \quad[0.01-0.05] ; \diamond[0.05-0.1] ; \diamond[0.1-0.2]$; $[0.2-1] ; \diamond[1-2] ; \diamond[2-5] ;$ and $\diamond>5$.

\section{References}

(1) Domańska, U.; Marciniak, A. Activity Coefficients at Infinite Dilution Measurements for Organic Solutes and Water in the Ionic Liquid 1-Ethyl-3methylimidazolium Trifluoroacetate. J. Phys. Chem. B 2007, 111, 11984-11988.

(2) Domanska, U.; Marciniak, A. Measurements of activity coefficients at infinite dilution of aromatic and aliphatic hydrocarbons, alcohols, and water in the new ionic liquid [EMIM] [SCN] using GLC. J. Chem. Thermodyn. 2008, 40, 860-866.

(3) Klamt, A.; Eckert, F. COSMO-RS: A Novel and Efficient Method for the A Priori Prediction of Thermophysical Data of Liquids. Fluid Phase Equilib. 2000, $172,43-72$. 
(4) Klamt, A. COSMO-RS From Quantum Chemistry to Fluid Phase Thermodynamics and Drug Design; Elsevier: Amsterdam, The Netherlands, 2005.

(5) Diedenhofen, M.; Eckert, F.; Klamt, A. Prediction of Infinite Dilution Activity Coefficients of Organic Compounds in Ionic Liquids Using COSMO-RS. J. Chem. Eng. Data 2003, 48, 475-479.

(6) University of Karlsruhe and Forschungszentrum Karlsruhe GmbH, TURBOMOLE V6.1 2009, 1989-2007, $25 \mathrm{GmbH}$, since 2007; available from http://www.turbomole.com.

(7) Everett, D. H. Effect of gas imperfection on G.L.C. measurements : a refined method for determining activity coefficients and second virial coefficients. Trans. Faraday Soc. 1965, 61, 1637-1645.

(8) Cruickshank, A. J. B.; Gainey, B. W.; Hicks, C. P.; Letcher, T. M.; Moody, R. W.; Young, C. L. Gas-liquid chromatographic determination of cross-term second virial coefficients using glycerol. Benzene + nitrogen and benzene + carbon dioxide at $50^{\circ} \mathrm{C}$. Trans. Faraday Soc. 1969, 65, 1014-1031.

(9) Grant, D. W. Gas-Liquid Chromatography; van Nostrand Reihold: London, UK, 1971.

(10) Poling, B. E.; Prausnitz, J. M. Properties of Gases and Liquids; McGraw-Hill Publishing, 2001.

(11) Design Institute for Physical Properties, Sponsored by AIChE. (2005; 2008; 2009; 2010; 2011; 2012). DIPPR Project 801 - Full Version. Design Institute for Physical Property Research/AIChE. Online version available at: http://app.knovel.com/hotlink/toc/id:

(12) Domańska, U.; Marciniak, A. Physicochemical Properties and Activity Coefficients at Infinite Dilution for Organic Solutes and Water in the Ionic Liquid 1-Decyl-3-methylimidazolium Tetracyanoborate. J. Phys. Chem. B 2010, $114,16542-16547$.

(13) Kato, R.; Gmehling, J. Activity coefficients at infinite dilution of various solutes in the ionic liquids $[\mathrm{MMIM}]^{+}\left[\mathrm{CH}_{3} \mathrm{SO}_{4}\right]^{-}, \quad[\mathrm{MMIM}]^{+}\left[\mathrm{CH}_{3} \mathrm{OC}_{2} \mathrm{H}_{4} \mathrm{SO}_{4}\right]^{-}$, $[\mathrm{MMIM}]^{+}\left[\left(\mathrm{CH}_{3}\right)_{2} \mathrm{PO}_{4}\right]^{-}, \quad\left[\mathrm{C}_{5} \mathrm{H}_{5} \mathrm{NC}_{2} \mathrm{H}_{5}\right]^{+}\left[\left(\mathrm{CF}_{3} \mathrm{SO}_{2}\right)_{2} \mathrm{~N}\right]^{-} \quad$ and $\left[\mathrm{C}_{5} \mathrm{H}_{5} \mathrm{NH}\right]^{+}\left[\mathrm{C}_{2} \mathrm{H}_{5} \mathrm{OC}_{2} \mathrm{H}_{4} \mathrm{OSO}_{3}\right]^{-}$. Fluid Phase Equilib. 2004, 226, 37-44.

(14) Štejfa, V.; Fulem, M.; Růžička, K.; Červinka, C.; Rocha, M. A. A.; Santos, L. M. N. B. F.; Schröder, B. Thermodynamic study of selected monoterpenes. J. Chem. Thermodyn. 2013, 60, 117-125.

(15) Štejfa, V.; Fulem, M.; Růžička, K.; Červinka, C. Thermodynamic study of selected monoterpenes II. J. Chem. Thermodyn. 2014, 79, 272-279. 
(16) Batiu, I. Vapor-liquid equilibria in the binary systems $n$-decane $+(-)$-menthone and n-decane $+(+)$-fenchone at temperatures between 344.45 and $390.75 \mathrm{~K}$. Fluid Phase Equilib. 2002, 198, 111-121.

(17) Yaws, C. L.; Narasimhan, P. K.; Gabbula, C. Yaws' Handbook of Antoine Coefficients for Vapor Pressure (2nd Electronic Edition); Knovel, 2009.

(18) Landolt-Börnstein - Group IV Physical Chemistry, Volume 20B - Vapor Pressure and Antoine Constants for Oxygen Containing Organic Compounds; Hall, K. R., Ed.; Springer, 2000.

(19) Wypych, George. (2008; 2012). Knovel Solvents - A Properties Database. ChemTec Publishing. Online version available at: http://app.knovel.com/hotlink/toc/id:kpKSAPD005/knovel-solventsproperties/knovel-solvents-properties.

(20) Štejfa, V.; Fulem, M.; Růžička, K.; Červinka, C. Thermodynamic study of selected monoterpenes III. J. Chem. Thermodyn. 2014, 79, 280-289.

(21) Sinnott, R.K.. (2005). Coulson and Richardson's Chemical Engineering Volume 6 - Chemical Engineering Design (4th Edition). Elsevier. Online version available at: http://app.knovel.com/hotlink/toc/id:kpCRCEVCE2/coulsonrichardsons-chemical/coulson-richards.

(22) Hazra, A.; Dollimore, D.; Alexander, K. Thermal analysis of the evaporation of compounds used in aromatherapy using thermogravimetry. Thermochim. Acta 2002, 392-393, 221-229.

(23) Fichan, I.; Larroche, C.; Gros, J. B. Water Solubility, Vapor Pressure, and Activity Coefficients of Terpenes and Terpenoids. J. Chem. Eng. Data 1998, 44, $56-62$.

(24) van Roon, A.; Parsons, J. R.; Govers, H. A. J.; Roon, A. v.; Parsons, J. R.; Govers, H. A. J. Gas chromatographic determination of vapour pressure and related thermodynamic properties of monoterpenes and biogenically related compounds. J. Chromatogr. A 2002, 955, 105-115.

(25) Aldrich Chemical Company Inc., Catalog Handbook of Fine Chemicals, Milwaukee WI, 1990, 1.

(26) Martins, M. A. R.; Pinho, S. P.; Coutinho, J. A. P. Critical Properties of Terpenes and Terpenoids. In Preparation.

(27) Joback, K. G.; Reid, R. C. Estimation of Pure-Component Properties from Group-Contributions. Chem. Eng. Commun. 1987, 57, 233-243.

(28) Joback, S. M. A Unified Approach to Physical Property Estimation Using Multivariate Statistical Techniques (doctoral dissertation), Cambridge, MA, 1984. 